\title{
Morbidities of preterm VLBW neonates and the bacteriological profile of sepsis cases
}

\author{
M M Hoque', A S M N U Ahmed², S K Halder ${ }^{3}$, M F H Khan", M A K A Chowdhury ${ }^{5}$
}

\begin{abstract}
Background: Preterm very low birth weight babies are at increased risk of perinatal, neonatal and postnatal mortality and morbidity, mainly due to infections and complications of prematurity. Mortality of VLBW neonates is 30 times more than that of normal weight. Outcomes of such infants have been reported extensively from developed countries, but less is known from developing countries like Bangladesh though prematurity is very common.
\end{abstract}

Objective: To determine the morbidities associated with preterm VLBW neonates with particular emphasis on sepsis.

Methods: A prospective cohort study was done in Special Care Baby Unit of a tertiary care teaching hospital from July 2009 to December 2009. Preterm VLBW neonates admitted within 7 days of age comprised the cohort for the study. Detailed physical findings and information on pregnancy, delivery and immediate postnatal period were recorded on enrolment. Sepsis workup was done whenever sepsis was suspected clinically. Daily follow-up was given till discharge/death and relevant clinical findings recorded. Data were analyzed using SPSS 12.

Results: A total of 738 neonates were admitted during study period, 92 were preterm VLBW and enrolled in the cohort. Fiftytwo $(56.5 \%)$ of the enrolled cases were male and $40(43.5 \%)$ female, male female ratio of 1.3:1. Mean gestational age was $30.8 \pm 2.4$ weeks and mean birth weight $1,320 \pm 133$ grams. Eight cases $(8.7 \%)$ had features of septicaemia on admission and 49 (53.2\%) neonates subsequently developed nosocomial infections. Other morbidities were jaundice (34.8\%), RDS (8.7\%), NEC (4.3\%), TTN (3.3\%), IVH (2.2\%) and PDA (1.1\%). Blood culture was positive in $29.8 \%$ among 57 suspected sepsis cases; Acinetobacter (41.2\%) was the most common organism, followed by Klebsiella pneumoniae (23.5\%), Escherichia coli (23.5\%) and Pseudomonas sp. (11.8\%). Sixty (62.5\%) preterm VLBW neonates were improved and discharged, 3 (3.3\%) cases were discharged on risk bond and $29(31.5 \%)$ died.

Conclusions: Preterm VLBW neonates are at increased risk of morbidity and mortality. Septicemia is the most common and devastating morbidity, most infections are hospital acquired. Therefore strict protocol for asepsis in neonatal units must be adhered to when handling these high risk infants.

Key words: Preterm, Very low birth weight, Septicaemia, Morbidity, Mortality.

Introduction: Between 1992 and 2008, globally under-five mortality rate have declined by two thirds, falling from 85 to 28 deaths per 1,000 live births; neonatal mortality dropped by one half during this period. ${ }^{1}$ In an article entitled 'Why are 4 million newborn babies dying each year?' Lawn et al. recognized that the greater part of the impressive reduction in infant mortality observed throughout the twentieth century was the result of reductions in post-neonatal mortality, neonatal mortality rates remained static. ${ }^{2}$ Among the neonates, very low birth weight (VLBW) preterm babies are at increased risk of perinatal, neonatal and postnatal mortality and morbidity, mainly due to infections and complications of prematurity. ${ }^{3,4}$ Mortality of VLBW neonates is 30 times more than that of newborns of normal weight. ${ }^{5}$ Despite constituting a small portion of LBW newborns, VLBW infants have a large impact on both neonatal mortality and morbidity. They demand high technology health care delivery and consume a great amount of resources.

Although outcomes of VLBW infants have been reported extensively from industrialized countries, ${ }^{6}$ less is known from the developing countries. Its prevalence is directly correlated with the developmental state of a country and is associated with poverty. Bangladesh is a developing country and an estimated $22 \%$ of babies are born prematurely and have low birth weight (LBW), ${ }^{7}$ but no such data is available for VLBW cases. Like any other part of the developing world, in Bangladesh also there has been a substantial reduction in under-five and infant mortality, but still having high neonatal mortality rates. In Bangladesh, survey in 2007 reported that the neonatal mortality (37/1,000 live births) accounts for about two-thirds of infant deaths (52/1,000 live births) and about half of deaths among children aged under 5 years (65/1,000 live births). ${ }^{8}$ The Bangladesh Demographic and Health Surveys indicate that the neonatal mortality rate declined in the early 1990s, but remained at $41-42$ between 1995-1999 and 1999-2003.9-11 Preterm VLBW babies are especially vulnerable because of immaturity of structures and functions of various systems. Well thermal control, monitoring of heart rate and respiratory rate, oxygen therapy, maintenance of fluid and electrolyte balance, special attention to nutritional support and safeguard against infection are the corner stone of management of these neonates. ${ }^{12}$ The use of intensive care facilities has been successful in decreasing the mortality rates of premature infants. However, with increasing survival, an increase in morbidities is encountered. Preterm VLBW neonates are vulnerable to develop respiratory distress syndrome (RDS), septicaemia, necrotizing enterocolitis (NEC), patent ductus arteriosus (PDA), intraventricular haemorrhage (IVH), and long-term sequelae such as threshold retinopathy of prematurity (ROP), chronic lung disease (CLD) and 


\section{Morbidities of preterm}

developmental disabilities..$^{13}$ Among the morbidities, sepsis is the most common in developing countries compared to the developed world. ${ }^{14}$ Smaller infants with lower gestational ages are also prone to invasive clinical interventions which result in higher incidence of nosocomial infections. Gram negative bacteria are mostly responsible for sepsis in this age group in developing countries. ${ }^{15-17}$ However, the organism pattern is different in developed and developing countries, and within a geographical location also may change with time. ${ }^{16}$

The purpose of the present study was to report the morbidities associated with preterm VLBW infants with particular emphasis on sepsis. Identification of bacterial etiology of sepsis in a tertiary care hospital will enable the physicians to treat these babies more rationally and thereby to reduce the morbidity and mortality of VLBW babies.

\section{Materials and methods:}

Study site: This prospective cohort study was conducted in the Special Care Baby Unit (SCABU) of Dhaka Shishu Hospital (DSH) from 1st July 2009 to 30th December 2009. $\mathrm{DSH}$ is the largest pediatric hospital in Bangladesh for primary and tertiary care. The SCABU has 30 neonatal beds and operates at full capacity at nearly all times.

Study Population: Newborn infants weighing $<1,500$ grams, gestational age $<35$ weeks and age $<7$ days, admitted during this period in SCABU were eligible for enrolment. Weight was measured on admission using a baby scale of 50 gm variation. Gestational age was determined on the basis of maternal dates (time from the first day of the last menstrual period) and further confirmed by New Ballard score. ${ }^{18}$

Patient evaluation: On admission, detailed history was taken about pregnancy, delivery and immediate postnatal period for each subject by interviewing the parents and thorough clinical examination was conducted by one of the investigators and findings were recorded in a structured questionnaire. The parents of the neonates were explained about the study and then witnessed verbal consent was taken. After enrolment, all neonates with suspected septicaemia underwent the following diagnostic procedures: complete blood count $(\mathrm{CBC}), \mathrm{C}$ - reactive protein (CRP) and blood culture. To diagnose other morbidities, CSF study (cytology, biochemistry and culture), serum bilirubin, x-ray chest, ultrasonography of brain, echocardiography and other relevant investigations were done as indicated. All neonates included into the cohort were closely followed during their hospital stay for clinical signs of infection. Sepsis workup was done whenever sepsis was suspected clinically. A patient was leveled as having septicaemia when, any two of the following signs and symptoms were present: lethargy, fever, hypothermia, recurrent apnea, bradycardia, abdominal distension, bleeding per rectal or gastric aspirate; plus any two of the following investigations were positive: blood culture, leukocytosis (total leukocyte count $>20,000 / \mathrm{cu} \mathrm{mm}$ of blood), leucopenia (total leukocyte count $>5,000 / \mathrm{cu} \mathrm{mm}$ of blood), thrombocytopenia (platelet count $<100,000 / \mathrm{cu}$ mm of blood), positive C-reacting protein ( $>10 \mathrm{mg} / \mathrm{L})$.

Patient management: All babies were managed by the unit consultant with their own protocol. Other than antibiotic therapy for sepsis cases, supportive therapy such as incubator care, correction of acidosis, maintenance of fluid and electrolyte balance, ventilatory assistance, phototherapy and blood transfusion was given as required. Investigators followed up all neonates daily till their discharge/death and recorded relevant clinical findings. The day of development of septicaemia was recorded.

Statistical analysis: The data were subjected to statistical analysis according to standard procedure. SPSS version 12.0 for Windows (SPSS Inc, Chicago, IL, USA) software was used for data recording and analysis.

Results: A total of 738 neonates were admitted in the SCBU during 1st July to 30th December 2009, 92 among them were preterm very low birth weight babies and enrolled in this study. Among the VLBW neonates, 60 (65\%) were admitted within 24 hours of birth and rest admitted between 24 hours and 7 days of birth. Fifty-two (56.5\%) of the enrolled cases were male and $40(43.5 \%)$ were female, male female ratio of $1.3: 1$. Their mean gestational age was $30.8 \pm 2.4$ weeks (ranged from 27-34 weeks) and mean birth weight of $1,320 \pm 133$ grams (ranged from 1,030-1,500 grams).

Out of 92 cases, 51 (55.4\%) VLBW babies were admitted only due to prematurity, rest $41(44.6 \%)$ had other complications along with prematurity, like suspected RDS $(20.7 \%)$, perinatal asphyxia $(10.9 \%)$, sepsis $(8.7 \%)$ and jaundice $(4.3 \%)$.

Table I: Causes of admission of the VLBW neonates $(\mathrm{n}=92)$

\begin{tabular}{|l|c|c|}
\hline Diseases on admission & Frequency & Percentage \\
\hline Suspected RDS & 19 & 20.7 \\
\hline Perinatal asphyxia & 10 & 10.9 \\
\hline Neonatal sepsis & 8 & 8.7 \\
\hline Neonatal jaundice & 4 & 4.3 \\
\hline Prematurity only & 51 & 55.4 \\
\hline Total & 92 & 100 \\
\hline
\end{tabular}




\section{Morbidities of preterm}

Other than 8 cases $(8.7 \%)$ that had features of septicaemia on admission, 49 (53.2\%) neonates subsequently developed septicaemia during hospital stay (nosocomial infections).

Figure I: Distribution of septicaemia of the VLBW neonates $(\mathrm{n}=92)$

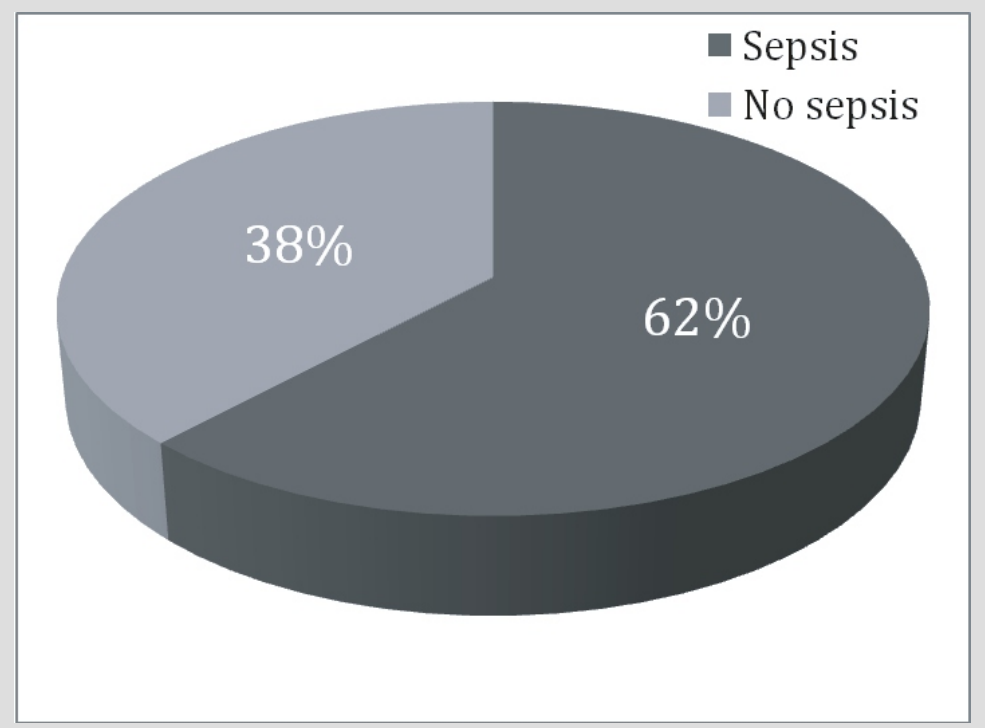

So incidence of septicaemia in preterm VLBW neonates in this study was $62 \%$. In addition to increased incidence of septicaemia among the enrolled VLBW neonates, there were other morbidities including jaundice (34.8\%), RDS (8.7\%), NEC (4.3\%), TTN (3.3\%), IVH (2.2\%) and PDA (1.1\%).

Figure II: Morbidities of the VLBW neonates during hospital stay ( $\mathrm{n}=92$ )

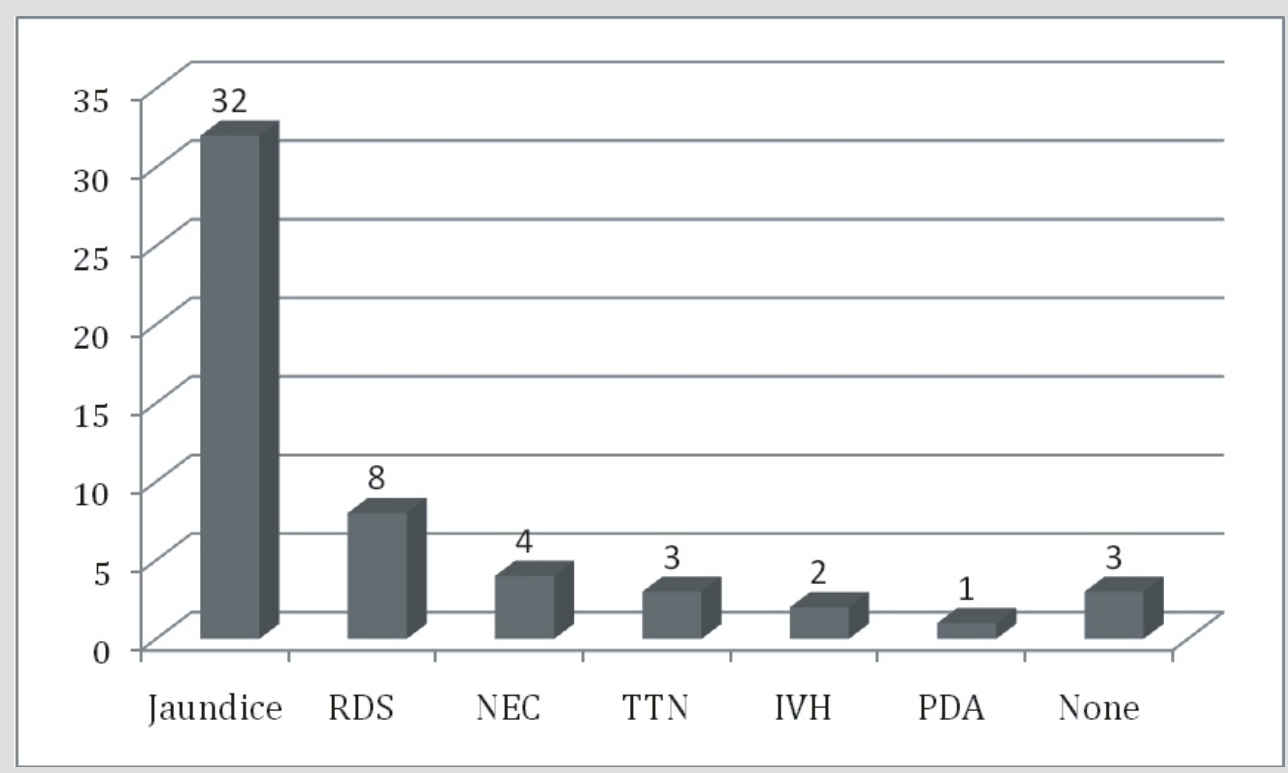




\section{Morbidities of preterm}

The above complications were present alone or in combination with septicaemia.

Among the laboratory investigations, CRP was positive in $86 \%$ cases and thrombocytopenia was found in $80.7 \%$ cases. Leukopenia (52.6\%) was more common than leukocytosis $(8.8 \%)$.

Table II: Laboratory findings of septicaemia cases $(n=57)$

\begin{tabular}{|l|c|c|}
\hline Name of investigations & Frequency & Percentage \\
\hline Blood culture positive & 17 & 29.8 \\
\hline Leukocytosis (TLC $>20,000)$ & 5 & 8.8 \\
\hline Leukopenia (TLC $<5,000)$ & 30 & 52.6 \\
\hline Thrombocytopenia (Platelets $<100,000)$ & 46 & 80.7 \\
\hline CRP positive $(>10 \mathrm{mg} / \mathrm{L})$ & 49 & 86.0 \\
\hline
\end{tabular}

Sixty $(65.2 \%)$ preterm very low birth weight neonates were improved and discharged, $3(3.3 \%)$ patients were discharged on risk bond and $29(31.5 \%)$ died.

Discussion: A total 92 patients were studied during the study period. Sixty $(65.2 \%)$ neonates were admitted within 24 hours and $32(34.8 \%)$ were admitted after 24 hours up to 7 days of age. There is no obstetric unit attached to this hospital and cases are referred from different centers, so babies coming from a distance are admitted late. Fifty-seven percent were male and $43 \%$ female with male female ratio of $1.3: 1$. Male babies are more likely than females to develop septicaemia and this male preponderance is similar to other studies. ${ }^{19}$ Males are cared for more in our society, this may also be a cause of male preponderance.

Septicaemia occurred in 57(62\%) cases of VLBW neonates in this series. Very low birth weight infants develop 2.7 times more sepsis than other infants since their immune system and skin barrier are immature and they are exposed to many invasive diagnostic and therapeutic procedures ${ }^{20}$ but it was much higher than other similar studies. Banu et al. found septicaemia in $16.4 \%$ of preterm VLBW neonates. ${ }^{21}$ Nagar et al. in their study of small for gestational age babies found the incidence of neonatal infection is about $26.2 \%{ }^{22}$ In a multi center cohort study done by Stoll et al. showed that the incidence of culture positive early onset neonatal sepsis was $1.9 \%$ though $50 \%$ of the cohort had clinical sepsis and $25 \%$ had culture positive late onset neonatal sepsis. ${ }^{23}$

Majority $(49 / 57,86 \%)$ of the septicaemia were hospital acquired. Nosocomial infections are a common problem in neonatal wards and an important cause of mortality in developing countries. The reported incidence of nosocomial sepsis in neonates from India ranges from $15.4 \%$ to $37 \%{ }^{24,25}$ Risk factors for nosocomial sepsis include preterm very low birth weight neonates, overcrowding in nurseries, assisted ventilation and use of peripheral venous and umbilical vascular catheters. ${ }^{25}$ Bed capacity of the SCABU at DSH has been increased from 20 to 30 without improvement of manpower specially nurses and cleaning staffs and other auxiliary supports. In our series we studied only the preterm VLBW babies and overcrowding at the nursery was responsible for this high nosocomial sepsis.

Among the 57 neonates diagnosed as septicaemia, 17 (29.8\%) had positive blood culture. Culture positivity rate was little lower than another of Ahmed ASMNU et al. in 1998 at the same SCABU, that showed $35 \%$ culture positive among the suspected septicaemia cases. ${ }^{26}$ Like other studies from developing countries, ${ }^{15-17,26}$ gram negative organisms were predominant in this study. Acinetobacter $(41.2 \%)$ was the most common organism found followed by Klebsiella pneumoniae (23.5\%) and Escherichia coli (23.5\%), Pseudomonas sp. was found in $2(11.8 \%)$ cases.

In this series the mean age of development of septicaemia was $6.7 \pm 2.4$ days indicating late-onset septicaemia was more common than early-onset disease, in contrast to other reports in which early-onset septicaemia generally has been more 


\section{Morbidities of preterm}

common. ${ }^{27}$ Predominantly nosocomial origin of infection was the reason for late-onset sepsis. The mean duration of hospital stay in neonates with septicaemia was $13.57 \pm 5.39$ days compared to $10.88 \pm 4.02$ days neonates without septicemia. It demonstrates that septicemia increases duration of hospital stay, increase workload and morbidity of VLBW neonates.

Other than septicaemia, common morbidities in this series were jaundice, RDS, NEC, IVH and PDA in $34.8 \%, 8.7 \%$, $4.3 \%, 2.2 \%$ and $1.1 \%$ cases respectively. Jaundice was mostly physiological, aggravated by sepsis. Several studies have reported almost similar findings. ${ }^{1428}$ Almost one-third $(31.5 \%)$ of the enrolled cases in this study died. Mortality is high in preterm VLBW neonates and this finding is comparable to other studies. ${ }^{13,29}$

Conclusions: Preterm VLBW neonates are at risk for several morbidities, of which septicemia is the most common and devastating morbidity leading to high mortality. Most cases of septicaemia are hospital acquired. Therefore to keep the infection rates low, strict protocol for asepsis in neonatal units must be adhered to when handling these high risk infants. There is a need for continuous neonatal infection surveillance to generate information on neonatal nosocomial infection rates and risk factors in our setting, so that appropriate preventive strategies can be adopted.

\section{References:}

1. The State of the World's Children Special Edition, UNICEF; New York: November 2009. Available: http://www.unicef.org/publications/ index 51772.html. Accessed: 21 May 2010.

2. Lawn JE, Cousens S, Bhutta ZA, Darmstadt GL, Martines J, Paul V, et al Why are 4 million newborns babies dying each year? Lancet. 2004;364:399-401.

3. Kate F. Transitional care and the convalescing NICU graduate Roberton's Textbook of Neonatology. 4th ed. China: Elsevier Churchill Livingstone; 2005.p. 385.

4. Khanam S, Islam MN, Kawser CA. Maternal and Socioeconomic risk factors associated with how birth weight. Bangladesh J Child health. 1995; 19:112-6.

5. Kabir ARML, Kawsar CA, Shahidullah M, Hassan MQ, Talukder MQK Situation analysis of child health in Bangladesh. 1994. Bangladesh J Child Health. 1995;19:53-60.

6. Lemons JA, Bauer CR, Oh W, Korones SB, Papile LA, Stoll BJ, et al Very low birth weight outcomes of the National Institute of Child health and human development neonatal research network. January 1995 through December 1996. NICHD Neonatal Research Network. Pediatrics. 2001;107:E1.

7. The State of the World's Children. UNICEF; New York: December 2007 p. 118.

8. National Institute of Population Research and Training (NIPORT), Mitra and Associates, and Macro International. 2009. Bangladesh Demographic and Health Survey 2007. Dhaka, Bangladesh and Calverton, Maryland, USA: National Institute of Population Research and Training, Mitra and Associates, and Macro International. 2007. p. 136.9.National Institute of Population Research and Training.
Bangladesh Demographic and Health Survey 1996-1997. Dhaka, National Institute of Population Research and Training, 1997. p. 252.

10. National Institute of Population Research and Training. Bangladesh Demographic and Health Survey 1999-2000. Dhaka, National Institute of Population Research and Training, 2001.p. 280.

11. National Institute of Population Research and Training. Bangladesh Demographic and Health Survey 2004. Dhaka, National Institute of Population Research and Training, 2005. p. 339.

12. Gomella TL, Cunningham MD, Eyal FG, Zenk KE. Neonatology: management, procedures, on-call problems, diseases, and drugs. 5th edn. New York: McGraw Hill; 2004. pp. 77-101.

13. Boo NY. Outcome of very low birth weight neonates in a developing country: experience from a large Malaysian maternity hospital. Singapore Med J. 1992;33:33-7.

14. Poundle P, Budhathoki S, Srivastava MK. Maternal risk factors and morbidity pattern of very low birth weight infants: A NICU based study at eastern Nepal. J Nepal Pediatr Soc. 2008;29:59-66.

15. Haque MM, Ahmed ASMNU, Chowdhury MAKA, Darmstadt GL, Saha SK. Septicaemic neonates without lumber puncture: what are we missing? J Trop Paediatr. 2006;52:63-5.

16. Bhutta ZA, Naqvi SH, Muzaffar T, Farooqui J. Neonatal sepsis in Pakistan: Presentation and pathogen. Acta Paediat Scand. 1991;80:596601.

17. Kapoor L, Randhawa VS, Deb M. Microbiological profile of neonatal septicemia in a pediatric care hospital in Delhi. J Commun Dis. 2005;37:227-32.

18. Ballard J, Khoury J, Wedig K, Wang L, Ellers-Walsman B, Lipp R. New ballard score expanded to include extremely premature infants. J Pediatr. 1991;119:417-23.

19. WHO young infants study group. Bacterial etiology of serious infections in young infants in developing countries: results of a multi centre study. Pediatr Infect Dis J. 1998;18 (suppl): S17-S22.

20. Makhoul IR, Sujov P, Smolkin T, Lusky A, Reichman B. Israel Neonatal Network. Pathogen-specific early mortality in very low birth weight infants with late-onset sepsis: a national survey. Clin Infect Dis. 2005:40:218-24

21. Banu K, Rahman S. Disease pattern in neonatal period. Bangladesh J Child Health. 1982;6:129-34.

22. Nagar A, Chowdhury MK, Kumar P. Small for gestational age babies: Indian scene. Indian J Pediatr. 1997;64:221-4.

23. Stoll BJ, Gordon T, Korones SB, Shankeran S, Tyson JE, Bauer CR et al. Early onset and late onset neonatal sepsis in VLBW neonates: a report from the National Institute of Child Health and Human Development Neonatal Research Network. J Pediatr. 1996;129:63-80.

24. Pawa AK, Ramji S, Prakash K, Thirupuram S. Neonatal nosocomial infection: profile and risk factors. Ind Pediatr. 1997;34:297-302.

25. Gupta P, Murali MV, Faridi MMA. Clinical profile of klebsiella septicemia in neonates. Indian J Pediatr. 1993;60:565-72.

26. Ahmed ASMNU, Chowdhury MAKA, Hoque MM, Darmstadt GL. Clinical and bacteriological profile of neonatal septicemia in a tertiary level pediatric hospital in Bangladesh. Ind Pediatr. 2002;39:1034-9.

27. Glandstone IM, Ehrenkranz RA, Edberg SC, Baltimore RS. A ten-year review of neonatal sepsis and comparison with the previous fifty year experience. Paediat Infect Dis J. 1990;9:819-25.

28. Rees CM, Eaton S, Pierro A. Trends in infant mortality from necrotising enterocolitis in England and Wales and the USA. Arch Dis Child Fetal Neonatal Ed. 2008;93:395-6.

29. Pereira SMP, Cardoso MHCA, Mattos H, Rozembaum R, Ferreira IV, Portinho MA et al. Sepsis-related mortality of very low birth weight Brazilian infants: the role of pseudomonas aeruginosa. International Journal of Pediatrics. vol;2009, Article ID 427682, 6 pages. doi: $10.1155 / 2009 / 427682$ 